\title{
475241 - ISOCAPNIC HYPERPNOEA AND RECOVERY TIME AFTER ANESTHESIA WITH ISOFLURANE
}

\author{
Rita Katznelson, MD ${ }^{1}$, Adriaan Van Rensburg, MD $^{1}$, Leonid Minkovich, MD $^{1}$, Zeev \\ Friedman, MD $^{2}$, Marcin Wasowicz, MD, PHD ${ }^{1}$, Joseph Fisher, MD $^{1}$ \\ 1. Anesthesia, Toronto General Hospital, Toronto, ON, Canada \\ 2. Anesthesia, Mt. Sinai Hospital, Toronto, ON, Canada
}

Introduction: Isocapnic Hyperpnoea (IH) is a method that increases alveolar ventilation while maintaining isocapnia. IH has been shown to accelerate awakening after short ambulatory anesthesia with isoflurane (1) and decrease recovery times in the operating room (OR) and post anesthesia care unit (PACU) after gynecological procedures with sevoflurane anesthesia. We conducted a prospective controlled clinical trial comparing recovery times from anesthesia with isoflurane in patients undergoing gynecological procedures who were randomized to either IH or Control groups.

Methods: After REB approval an informed consent was obtained from 19 patients scheduled for elective gynecological surgery. No premedication was given. Induction of anesthesia was standardized to propofol and fentanyl. Endotracheal intubation was facilitated with rocuronium. Normocapnic mechanical ventilation was achieved with air/oxygen mixture and the fresh gas flow of $1 \mathrm{~L} / \mathrm{min}$. Maintenance of anesthesia included iosoflurane and fentanyl. At the end of surgery isoflurane anesthesia was discontinued in both groups. After turning off the isoflurane vaporizer patients were randomized into either IH or control groups. IH group received 2-3 times their intraoperative minute ventilation with a non-rebreathing system while maintaining isocapnia. Control patients were allowed to recover from anesthesia in the conventional way. We recorded the time intervals from turning off the isoflurane vaporizer to a) resumption of spontaneous ventilation, b) eye-opening to verbal command, c) extubation, d) readiness to leave the OR, e) the time that first postoperative analgesic medication was given, and f) readiness for PACU discharge.

Results: A total of 10 patients were randomized to the IH group and 9 to the control group. There was no difference with respect to age and BMI between the $\mathrm{IH}$ and control groups respectively.(Age, $50 \pm 13.0$ vs. $49 \pm 10.5$ years, p=0.7; BMI, $26.8 \pm 6.5$ vs. 28.8 $\pm 8 \mathrm{~kg} / \mathrm{m} 2, \mathrm{p}=0.6$ ) The times from discontinuing isoflurane anesthesia to eye-opening, extubation, and readiness for PACU discharge were significantly shorter in the IH group.(Table 1)

Discussion: IH accelerates recovery from isoflurane anesthesia and shortens PACU length of stay.

References: 1)Br J Anaesth 2003; 91(6):787-792.

Comparison of revcovery times and length of PACU stay after isoflurane anesthesia in the IH and Control groups 


\begin{tabular}{|c|c|c|c|}
\hline & $\begin{array}{c}\text { IH Group } \\
(\mathrm{n}=10)\end{array}$ & $\begin{array}{c}\text { Control Group } \\
(\mathrm{n}=9)\end{array}$ & P value \\
\hline Resumption of spontaneous ventilation (min) & $3.5 \pm 2.0$ & $6.3 \pm 3.3$ & 0.06 \\
\hline Opening eyes in response to verbal command & $5.8 \pm 1.4$ & $13.2 \pm 4.1$ & 0.01 \\
\hline Time of Extubation (min) & $6.3 \pm 1.6$ & $13.9 \pm 3.9$ & 0.01 \\
\hline Fulfill criteria for leaving OR (min) & $8.2 \pm 1.5$ & $17.0 \pm 4.6$ & 0.05 \\
\hline Time of first postoperative pain medication (min) & $33 \pm 5.1$ & $43 \pm 12.4$ & 0.06 \\
\hline PACU discharge (min) & $75 \pm 15.6$ & $97.0 \pm 26.0$ & 0.03 \\
\hline
\end{tabular}

